\title{
Universal non-equilibrium properties of dissipative Rydberg gases
}

\author{
Matteo Marcuzzi, ${ }^{1}$ Emanuele Levi, ${ }^{1}$ Sebastian Diehl, ${ }^{2}$ Juan P. Garrahan, ${ }^{1}$ and Igor Lesanovsky ${ }^{1}$ \\ ${ }^{1}$ School of Physics and Astronomy, University of Nottingham, Nottingham, NG7 2RD, United Kingdom \\ ${ }^{2}$ Institute for Theoretical Physics, University of Innsbruck, A-6020 Innsbruck, Austria
}

\begin{abstract}
We investigate the out-of-equilibrium behavior of a dissipative gas of Rydberg atoms that features a dynamical transition between two stationary states characterized by different excitation densities. We determine the structure and properties of the phase diagram and identify the universality class of the transition, both for the statics and the dynamics. We show that the proper dynamical order parameter is in fact not the excitation density and find evidence that the dynamical transition is in the "model A" universality class, i.e. it features a non-trivial $\mathbb{Z}_{2}$ symmetry and a dynamics with non-conserved order parameter. This sheds light on some relevant and observable aspects of dynamical transitions in Rydberg gases. In particular it permits a quantitative understanding of a recent experiment [C. Carr et al., Phys. Rev. Lett. 111, 113901 (2013)] which observed bistable behavior as well as power-law scaling of the relaxation time. The latter emerges not due to critical slowing down in the vicinity of a second order transition, but from the non-equilibrium dynamics near a so-called spinodal line.
\end{abstract}

PACS numbers: 64.60.Ht, 64.60.My, 05.30.-d, 32.80.Ee

Introduction. - The study of the emergence of collective behavior in many-body systems represents a very active field of research. Fundamental insights, such as the onset of universality and its consequences [1-3] are central for our understanding of matter in general. In recent years, there has been a growing interest in understanding dynamical phase transitions [4-6] in the context of driven open many-body quantum systems [7-21], and progress in the manipulation of ultracold atoms [22] has made it possible to access and explore many-body phenomena under precisely controllable experimental conditions [23-27].

In this context, a class of systems that offers a rich and intricate physics is represented by so-called Rydberg gases [28-33], i.e., atomic clouds in which atoms are laser-excited to high-lying energy levels. The main consequence of the population of such orbitals is a considerable increase $[29,30]$ in the interaction strength. This is at the heart of several non-trivial dynamical phenomena, both for closed systems undergoing coherent evolution and showing enhanced spatial (anti-)correlations [34-37], and for open ones, in which the interplay between driving and dissipation leads instead to intermittency [38], glassy behavior [39] and bistable behavior [40].

The dissipative case has been recently studied via a mean-field approach [41-43], numerical calculations in one dimension [44-46] and an approximate rate equation description in higher dimensions [33, 47-49]. These investigations highlighted the presence of various stationary regimes and the existence of first and second order phase transitions. In addition, experiments have probed the static and dynamic features of these systems revealing bimodal behaviors [31] and optical bistabilities [32].

The aim of this work is to shed light on the bistable transition in a dissipative Rydberg gas with particular focus on its dynamics and to connect the findings to recent observations. For the stationary state, the transition is related to the spontaneous breaking of a $\mathbb{Z}_{2}$ symmetry and falls into the Ising universality class. The effective static order parameter is an appropriately shifted Rydberg excitation density. The dynamics is found to be of "model A" type according to the standard classification of Ref. [4]. This means that its critical properties coincide with those of a classical stochastic process described by a Langevin equation governing the interplay between a conservative, $\mathbb{Z}_{2}$-preserving force and white Gaussian noise. This corresponds to an Ising model subject to a spin-flipping dynamics which does not preserve the total magnetization, i.e., Glauber dynamics. However, within the dynamical framework it becomes clear that the dynamical order parameter is not formally identical to the Rydberg excitation density and the $\mathbb{Z}_{2}$ symmetry identified in the static case must be non-trivially generalized. Linking to recent experimental studies [32], we note that the dynamic transitions observed there take in fact place near the so-called spinodal lines of the mean-field phase diagram. This kind of regime has already been studied from a dynamical perspective in the context of non-interacting atoms in optical cavities ("Dicke model" scenario) [50], Josephson junctions [51] and carefullyengineered micromechanical oscillators [52], which fall into the same universality class. The connection established to "model A" physics allows us to extract a universal scaling law for relaxation times for which quantitative agreement with experiment is found. We believe that this perspective will be useful for analyzing and understanding the dynamical phenomena observed in other related experiments, such as the one presented in Ref. [31].

The model. - We employ the standard description of a Rydberg gas in terms of (fictitious) interacting spin$1 / 2$ particles $[29,40,41,53]$, where the states $|\downarrow\rangle$ and $|\uparrow\rangle$ correspond to the atomic ground and Rydberg states re- 
spectively. The dynamics of the system's density matrix $\widehat{\rho}$ is governed by the quantum master equation (QME) $\partial_{t} \widehat{\rho}=-i[H, \widehat{\rho}]+\left(\mathcal{L}_{1}+\mathcal{L}_{2}\right)[\widehat{\rho}][54]$ with Hamiltonian

$$
H=\Omega \sum_{k} \widehat{\sigma}_{k}^{x}+\Delta \sum_{k} \widehat{n}_{k}+\sum_{k \neq p} V_{k p} \widehat{n}_{k} \widehat{n}_{p}
$$

expressed in a frame rotating at the laser frequency [55]. Here $\Omega$ is the (real) Rabi frequency and $\Delta$ the detuning of the excitation laser with respect to the ground state - Rydberg state transition. The (symmetric) interaction between two atoms positioned at $\vec{r}_{k}$ and $\vec{r}_{p}$ is typically isotropic and of van der Waals type $2 V_{k p}=$ $V_{k p}+V_{p k}=C_{6}\left|\vec{r}_{k}-\vec{r}_{p}\right|^{-6}[29,30]$, although other potentials may arise in different regimes [56-58]. Note, the our treatment merely requires the interactions to be shortranged. Finally, we have defined the excitation density $\widehat{n}_{k}=\left(\mathbb{1}_{k}+\widehat{\sigma}_{k}^{z}\right) / 2$, with $\left\{\widehat{\sigma}_{k}^{x}, \widehat{\sigma}_{k}^{y}, \widehat{\sigma}_{k}^{z}\right\}$ being the usual quantum spin operators acting on the $k$-th site.

Relating to previous experimental and theoretical studies [29, 31, 39-42, 53, 59], dissipation is described by a dissipator of Lindblad form

$$
\mathcal{L}_{j}[(\cdot)]=\sum_{k}\left[L_{j k}(\cdot) L_{j k}^{\dagger}-\frac{1}{2}\left\{L_{j k}^{\dagger} L_{j k},(\cdot)\right\}\right] .
$$

Such formulation is appropriate for ultra-cold quantum optical systems like ours where noise occurs through the coupling of the system's degrees of freedom to the radiation field that effectively acts as a memory-less bath. We account for two different mechanisms: One is independent atomic decay (at rate $\Gamma$ ) from the Rydberg state to the ground state, with jump operator $L_{1 k}=\sqrt{\Gamma} \widehat{\sigma}_{k}^{-}=$ $\sqrt{\Gamma}\left(\widehat{\sigma}_{k}^{x}-i \widehat{\sigma}_{k}^{y}\right) / 2$. The second one is dephasing of the Rydberg state relative to the ground state, occurring at rate $K$ with $L_{2 k}=\sqrt{K} \widehat{n}_{k}$.

Mean-field equations of motion.- A mean-field treatment of the Rydberg gas has been already conducted to some extent in other works, see, e.g., [41]; here we just briefly summarize the derivation of the equations of motion. We consider the complete set of one-atom observables $\left\{\mathbb{1}_{k}, \widehat{\sigma}_{k}^{x}, \widehat{\sigma}_{k}^{y}, \widehat{n}_{k}\right\}$ and calculate their respective averages $\{1, \vec{S}\} \equiv\left\{1, S^{x}, S^{y}, n\right\}$ according to $\langle(\cdot)\rangle=$ $\operatorname{tr}\{\widehat{\rho}(\cdot)\}$. Applying the QME, assuming spatial uniformity and factorising all quadratic expectations yields the closed set of dynamical equations

$$
\left\{\begin{array}{l}
\dot{S}^{x}=-(\Delta+V n) S^{y}-\frac{\Gamma+K}{2} S^{x} \\
\dot{S}^{y}=2 \Omega-4 \Omega n+(\Delta+V n) S^{x}-\frac{\Gamma+K}{2} S^{y} \\
\dot{n}=\Omega S^{y}-\Gamma n,
\end{array}\right.
$$

with $V=2 \sum_{p} V_{k p}$ the mean-field interaction energy.

Stationary regime. - Introducing the effective parameters

$$
a=2+\frac{1}{4} \frac{\Gamma(\Gamma+K)}{\Omega^{2}}, b=\left(\frac{V}{\Omega}\right)^{2} \frac{\Gamma}{\Gamma+K}, c=\frac{\Delta}{V}
$$
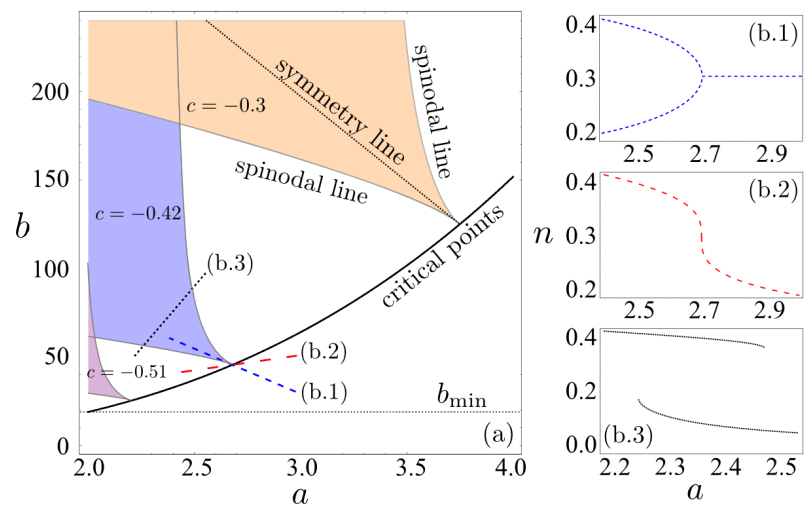

FIG. 1. (Color online) Phase diagram in the $a-b$ plane [as defined in Eq. (4)] for three different values of $c$. The shaded areas correspond to domains portraying three stationary real solutions. Their boundaries identify the spinodal lines. The black curve represents the path threaded by the critical point when varying $c$, corresponding to the projection of the critical line $\left\{a_{c}, b_{c}, c\right\}=\left\{-9 /(8 c),-27 /\left(8 c^{3}\right), c\right\}$ onto the $a-b$ plane. This line meets the vertical axis at $b_{\min }=512 / 27$. Panels (b) relate to $c=-0.42$ and show the excitation density $n$ taken along the three cuts shown in panel (a): in panels (b.1) and (b.2) we show $n$ as observed on the blue and red dashed lines, respectively, which correspond to the "thermal" and "magnetic" directions (see main text). The black dashed line which crosses the spinodal boundaries probes instead the stable-bistable transition, corresponding to the hysteresis-like profile in panel (b.3).

allows us to formulate the problem in a concise way. We can eliminate $S^{x}$ and $S^{y}$ from the stationary solutions of (3), thus obtaining an algebraic equation for the stationary average number of excitations $n$,

$$
n\left[a+b(c+n)^{2}\right]=1 .
$$

This expression is a cubic real polynomial in $n$ and admits from 1 to 3 real roots depending on the specific values taken by $(a, b, c)$ within the physically allowed space $\{a \geq 2, b \geq 0\}$. In Fig. 1 we report the corresponding phase diagram in the $a-b$ plane for different choices of $c$. The stable phase of the system corresponds to the parameter domain displaying only one acceptable solution. Complementary to this domain is the bistable regime [38, 40, 41], with Eq. (5) featuring three solutions, only two of which are stable. The boundaries between stable and bimodal regimes are the spinodal lines, where at least two solutions coincide. For any value of $c$, the spinodal lines coalesce into a critical point identified by $a_{c}=-9 /(8 c)$ and $b_{c}=-27 /\left(8 c^{3}\right)$, which corresponds to having three coincident real solutions for Eq. (5). By varying the laser detuning $\Delta$, this point moves along the curve $b=(4 a / 3)^{3}$ shown in Fig. 1 and lies within the aforementioned physical parameter space only when $-9 / 16 \leq c \leq 0$. Varying $\Omega$, instead, implies moving along straight lines departing from $(a=2, b=0)$ with a slope increasing with the interaction strength $V$. Thus, 
one can work out a threshold value (corresponding to the tangent to the critical curve) $V_{\min }=4(\Gamma+K)$ below which the transition cannot be found by just changing the laser parameters.

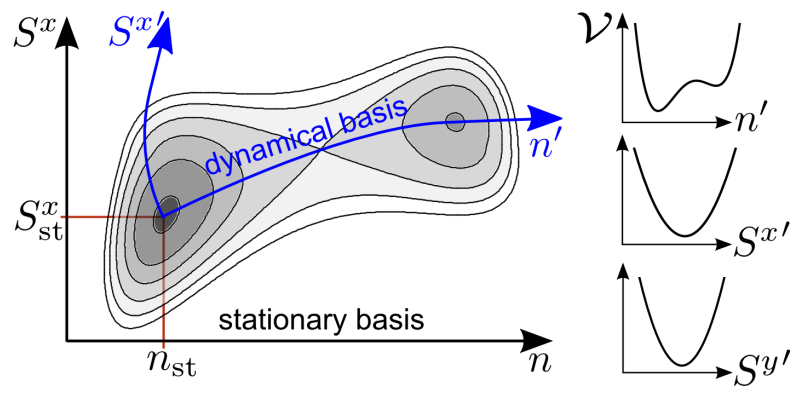

FIG. 2. (Color online) The non-linear transformation from the stationary basis of observables $\left\{S^{x}, S^{y}, n\right\}$ to the dynamical one $\left(\left\{S^{x^{\prime}}, S^{y^{\prime}}, n^{\prime}\right\}\right)$ is qualitatively depicted in the main panel. The critical/off-critical behavior of the $\delta S^{\prime}$ components can be captured by superimposing an effective potential $\mathcal{V}$ which we discuss later in the text. Crucially, the doublewell structure (responsible for the "model A" physics) is only felt by the critical $n^{\prime}$, whereas along the other "massive" directions the system only probes single quadratic wells which play no role in the transition, as we sketch on the right.

We investigate now the universal features near the critical point: We expand Eq. (5) to leading order in a perturbation of the parameters around their critical values $\left(a=a_{c}+\delta a\right.$ and $\left.b=b_{c}+\delta b\right)$ and study the corresponding variation of the stable solutions $n_{\mathrm{st}}=$ $n_{c}+\delta n=-2 c / 3+\delta n$. We identify a special direction $\delta b=\left(-9 / c^{2}\right) \delta a$ [in the following referred to as symmetry line, see Fig. 1(a)] along which the solution is invariant under the transformation $\delta n \rightarrow-\delta n$ (with a more complicated one holding for $S^{x}$ and $S^{y}$ ). Thus, a $\mathbb{Z}_{2}$ symmetry for the stationary value of the excitation density $n$ emerges, which is spontaneously broken in the bistable phase [see Fig. 1(b.1)]. When approaching the critical point along the symmetry line we find $\delta n \sim(-\delta a)^{1 / 2}$. For any other direction [e.g., the red dashed line in Fig. 1(a)] the system does not switch phases when crossing the critical point. The corresponding behavior, portrayed in Fig. 1(b.2), is described by $|\delta n| \sim|\delta a|^{1 / 3}$. We can thus conclude that this transition belongs to the (static) Ising universality class with order parameter $\delta n=n-n_{c}$ : In fact, the magnetization $m$ of an Ising model, as a function of the temperature $T$, the critical temperature $T_{c}$ and the magnetic field $h$ is known to obey $m(T, h=0) \sim\left|T-T_{c}\right|^{\beta}$ and $m\left(T_{c}, h\right) \sim h^{1 / \delta}$, with mean-field exponents $\beta=1 / 2$ and $\delta=3[3,60]$. In analogy, we associate the symmetry line (b.1) to the thermal direction and any deviation from it to the presence of a $\mathbb{Z}_{2}$-breaking magnetic field. Finally, a generic choice of the parameters will lead to probing the spinodal behavior shown in Fig. 1(b.3), which has indeed been highlighted in previous theoretical and experimental studies [32, 42].
Dynamical vs. static order parameter. - We now turn to the dynamical aspects: As a first step, we perform an analysis of the stability of the stationary points. In their neighborhood, we expand the r.h.s. of Eqs. (3) to linear order in the deviations (e.g., $\delta n=n-n_{\text {st }}$ ), which obey the differential equation $\delta \dot{\vec{S}}=M \delta \vec{S}$. The eigenvalues of the stability matrix $M$ constitute the rates of approach or escape from the stationary point. Whenever the solution is unique, it is stable as well; when three solutions are present, the two extremal ones are stable, while the one in the middle is unstable, cf. [41].

Here, however, we focus on the universal properties that emerge near the critical point and the spinodal lines, where null eigenvalues appear. This implies the emergence of (leading) algebraic decays $\delta n \sim t^{-1 / \zeta}$ towards stationarity, with different exponents in the spinodal $(\zeta=1)$ and critical $(\zeta=2)$ regimes. In the critical case, scaling arguments predict an algebraic law of the form $t^{-\beta /(\nu z)}$, with $z$ being the dynamical critical exponent. The determination of the static universality class (Ising) provides us with the mean-field exponents $\beta=\nu=1 / 2$. Thus, we conclude that $z=2$, describing the dynamics of a diffusive system.

In addition to the null direction, the stability matrix displays two non-vanishing ("massive") eigenvalues, identifying two non-critical directions. Hence, the effective order parameter for the long time dynamics has only one component $\delta n^{\prime}$; due to the non-linearity of Eqs. (3), $\delta n^{\prime}$ is a non-trivial function of the original variables (see axes in Fig. 2), which only coincides with $\delta n$ in the stationary regime. A discrete $\mathbb{Z}_{2}$ invariance of the equations of motion under $\delta n^{\prime} \rightarrow-\delta n^{\prime}$ is expected along the symmetry line of Fig. 1 . We analytically verified this up to the quadratic order. The absence of any apparent conservation law strongly suggests that the dynamics of the system at hand belongs to the (one component) "model A" universality class. We remark that this is similar to the critical point in the driven open Dicke model $[7,12-15]$, which however constitutes a zero dimensional model where the mean-field exponents are exact. Consistently with this picture, at the critical point the equation of motion reads $\delta \dot{n}^{\prime} \propto\left(\delta n^{\prime}\right)^{3}$ at leading order. In contrast, along the spinodal lines we find $\delta \dot{n}^{\prime} \propto\left(\delta n^{\prime}\right)^{2}$ and, consequently, an exponent $\zeta=1$. We remark that the emergent symmetry introduced above does not lie among those identified in Ref. [41] (i.e., $\left\{\Delta, V, S^{x}\right\} \rightarrow$ $\left\{-\Delta,-V,-S^{x}\right\}$ and $\left.\left\{\Omega, S^{x}, S^{y}\right\} \rightarrow\left\{-\Omega,-S^{x},-S^{y}\right\}\right)$, which are unbroken in both phases.

Metastable dynamics and connection to experiments. We now connect these findings to recent experiments $[31,32]$ that have investigated the dynamics of dissipative Rydberg gases. The work presented in Ref. [31] has explored the phase diagram in the $\Omega-\Delta$ plane, shown in Fig. 3(a). Another experiment [32] has high- 

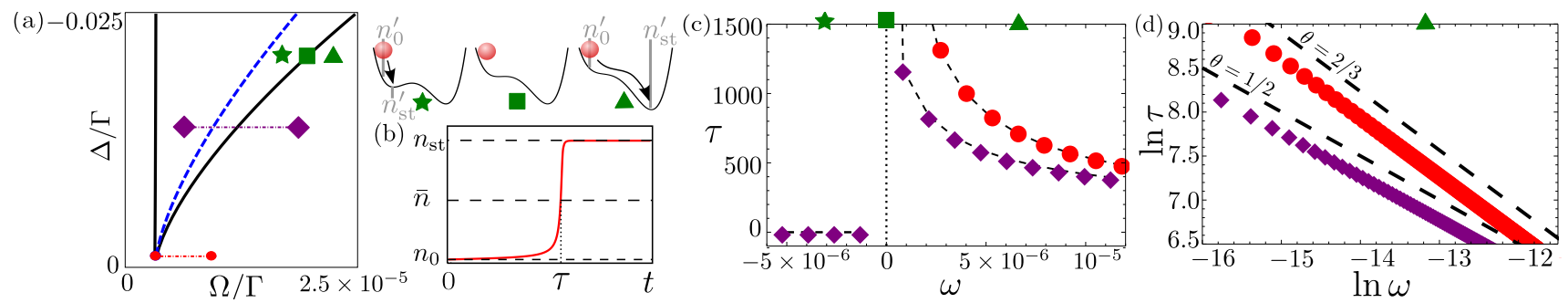

FIG. 3. (Color online) Emergence of metastable regimes in the vicinity of the spinodal lines. In panel (a) we show the phase diagram in the $\Delta / \Gamma-\Omega / \Gamma$ plane at $K=0$ and $V$ fixed. The solid and dashed curves show the spinodal and symmetry lines of Fig. 1, respectively. On the right we display the qualitative structure of the mean-field potential $\mathcal{V}\left(n^{\prime}\right)$ for parameters corresponding to inside $(\star)$, on $(\boldsymbol{\square})$ and outside $(\boldsymbol{\Delta})$ the spinodal lines (see text). In panel (b) we show an example for the relaxation of the excitation density $n$ (from an initial value $n_{0}$ ) towards the stationary value $n_{\text {st }}$, for parameters near one of these boundaries. Here we observe a metastable plateau whose lifetime $\tau$ is determined by the first crossing time of the midpoint $\bar{n}=\left(n_{\mathrm{st}}+n_{0}\right) / 2$. Panel (c, d) display a power-law divergence of $\tau$ as a function of the reduced Rabi frequency $\omega=\left(\Omega-\Omega_{c}\right) / \Omega_{c}$ varied along the red and purple curves in panel (a) [see for comparison the experimental data shown in Fig. 4 of Ref. [32]]. The power changes from $\theta=1 / 2$ in the bistable region [diamonds in panels (a,c,d)] to $\theta=2 / 3$ when intersecting the critical point [disks in panels $(\mathrm{a}, \mathrm{c}, \mathrm{d})]$.

lighted a bistable behavior similar to the one presented in Fig. 1(b.3). Moreover, a power-law behavior of the relaxation time close to a "critical value" of the excitation laser strength was reported.

In order to gain some intuitive insight on these phenomena, we exploit our knowledge of the universality class and introduce a phenomenological mean-field potential $\mathcal{V}\left(n^{\prime}\right)=\alpha n^{\prime}-\beta\left(n^{\prime}\right)^{2}+\gamma\left(n^{\prime}\right)^{4}$ which reflects the profile reported in the topmost panel on the r.h.s. of Fig. 2. The corresponding mean-field dynamics is given by $\dot{n}^{\prime}=-\partial_{n^{\prime}} \mathcal{V}\left(n^{\prime}\right)$. For fixed $\beta>0$ and $\gamma>0$, this equation portrays a stable (one minimum) to bistable (two minima) transition at a threshold value $\alpha_{c}=(2 \beta / 3)^{3 / 2} \gamma$. The experiments mentioned above are performed such that initially no excited atoms are present and subsequently the excitation laser is switched on at given values of $\Delta$ and $\Omega$. In the bistable phase $\left(\alpha<\alpha_{c}\right)$ this may lead to a fast relaxation towards the nearest local minimum of $\mathcal{V}\left(n^{\prime}\right)$, which is not necessarily the global one [see $(\star)$ in Fig. 3(a)]. Accounting for fluctuations (beyond mean-field) may in general introduce an additional time scale beyond which this picture is no longer valid and a different physics emerges. Furthermore, a correction to the value of the exponent would ensue. However, the agreement between our predictions and experimental observations highlighted below suggests that these features are quite robust (at least down to three dimensions) and that current experiments indeed probe this "short time physics".

When $\alpha=\alpha_{c}$, i.e., on a spinodal line, one of the minima becomes an inflection point [see (ם) in Fig. 3(a)]. For $\alpha \gtrsim \alpha_{c}$ [see ( $\left.\mathbf{\Lambda}\right)$ in Fig. 3(a)], in the proximity of the disappeared minimum one can identify a region of vanishing slope of the potential, which leads to a characteristic slow dynamics in a flat landscape. This is reflected in the evolution of observables by the appearance of long-lived plateaus (see, e.g., Fig. 3(b) and Fig. (4) in Ref. [32]) whose lifetime $\tau$ diverges when approaching $\alpha_{c}$. By analytically solving the phenomenological equations of motion one obtains $\tau \sim\left(\alpha-\alpha_{c}\right)^{-\theta}$ with $\theta=1 / 2$, which agrees with the experimental estimate $\theta=0.53 \pm 0.10$ of Ref. [32]. If, instead of a spinodal line, one crosses the critical point (i.e., $\beta=0$ in $V\left(n^{\prime}\right)$ ), a different exponent $\theta=2 / 3$ is found. It should be possible to test this prediction in the experimental setting of Ref. [32], which would constitute strong evidence for universal model A physics.

Note that, instead of $n^{\prime}$, the standard experimental observable is the excitation density $n$. Nonetheless, since the modes $\delta S_{x / y}^{\prime}$ decay exponentially fast, the long time dynamics is dominated by $n^{\prime}$ and critical scaling is exhibited by the standard observables as well. This and the results of the phenomenological model are confirmed by the numerical solution of the full dynamical equations (3): Mimicking the experimental procedure [61], i.e., computing $\tau$ for different values of $\Omega$ in the proximity of the spinodal lines while keeping $V, \Delta, \Gamma, K$ fixed [see Fig. 3(a)] indeed yields algebraic divergences $\tau \sim\left(\Omega-\Omega_{c}\right)^{-\theta}$ [see Figs. $3(\mathrm{c})$ and $(\mathrm{d})]$ with exponents $\theta \approx 0.5$ and $\theta \approx 0.66$ for the spinodal and critical cases, respectively.

Conclusions and outlook. - We have found strong evidence for the non-equilibrium dynamics of the dissipative Rydberg gas being governed by the much studied $[4,6,62-65]$ "model A" universality class whose lower critical dimension is two. This would exclude the presence of a phase transition in dimension one - a question that was raised by the authors of Ref. [42].

We moreover observed the emergence of a metastable regime in close proximity to the spinodal lines, whose lifetime diverges algebraically as observed in a recent experiment. Surprisingly, our mean-field approach is quantitatively accurate in determining the exponent of this 
power-law. Whether this is due to experimental uncertainties or a result of some more subtle issues arising in the spinodal regime constitutes a matter of future experimental and theoretical investigation.

Acknowledgements - The research leading to these results has received funding from the European Research Council under the European Union's Seventh Framework Programme (FP/2007-2013) / ERC Grant Agreement No. 335266 (ESCQUMA), the EU-FET Grant No. 512862 (HAIRS), the Austrian Science Fund (FWF) through the START grant Y 581-N16 and SFB FOQUS, Project No. F4006-N16. We also acknowledge financial support from EPSRC Grant no. EP/J009776/1.

[1] J. Zinn-Justin, Quantum Field Theory and Critical Phenomena, 4th ed., International Series of Monographs on Physics (Clarendon Press, 2002).

[2] S. Sachdev, Quantum Phase Transitions (Cambridge University Press, 1999).

[3] S.-K. Ma, Modern theory of critical phenomena, Frontiers in Physics (Reading, MA: W. A. Benjamin,, 1976).

[4] P. C. Hohenberg and B. I. Halperin, Rev. Mod. Phys. 49, 435 (1977).

[5] A. Kamenev, Field Theory of Non-Equilibrium Systems (Cambridge University Press, 2011).

[6] U. C. Täuber, Critical Dynamics - A field theory approach to equilibrium and non-equilibrium scaling behavior (Cambridge University Press, Cambridge, 2014).

[7] S. Safaei, Ö. E. Müstecaplıŏlu, and B. Tanatar, New Journal of Physics 15, 083037 (2013).

[8] S. Safaei, Ö. E. Müstecaplığlu, and B. Tanatar, Laser Physics 23, 035501 (2013).

[9] K. Baumann, C. Guerlin, F. Brennecke, and T. Esslinger, Nature 464, 1301 (2009).

[10] M. Heyl, A. Polkovnikov, and S. Kehrein, Phys. Rev. Lett. 110, 135704 (2013).

[11] I. Lesanovsky, M. van Horssen, M. Guţă, and J. P. Garrahan, Phys. Rev. Lett. 110, 150401 (2013).

[12] D. Nagy, G. Szirmai, and P. Domokos, Phys. Rev. A 84, 043637 (2011).

[13] B. Oztop, M. Bordyuh, Ö. E. Müstecaplığlu, and H. E. Tureci, New J. Phys. 14, 085011 (2012).

[14] E. G. d. Torre, S. Diehl, M. D. Lukin, S. Sachdev, and P. Strack, Phys. Rev. A 87, 023831 (2013).

[15] F. Brennecke, F. R. Mottl, K. Baumann, R. Landig, T. Donner, and T. Esslinger, PNAS 110, 11763 (2013).

[16] E. M. Kessler, G. Giedke, A. Imamoglu, S. F. Yelin, M. D. Lukin, and J. I. Cirac, Phys. Rev. A 86, 012116 (2012).

[17] M. Foss-Feig, K. R. A. Hazzard, J. J. Bollinger, and A. M. Rey, Phys. Rev. A 87, 042101 (2013).

[18] S. Diehl, A. Tomadin, A. Micheli, R. Fazio, and P. Zoller, Phys. Rev. Lett. 105, 015702 (2010).

[19] C. Karrasch and D. Schuricht, Phys. Rev. B 87, 195104 (2013).

[20] L. M. Sieberer, S. D. Huber, E. Altman, and S. Diehl, Phys. Rev. Lett. 110, 195301 (2013).

[21] U. C. Täuber and S. Diehl, Phys. Rev. X 4, 021010 (2014).
[22] I. Bloch, J. Dalibard, and W. Zwerger, Rev. Mod. Phys. 80, 885 (2008).

[23] M. Greiner, O. Mandel, T. Esslinger, T. W. Hänsch, and I. Bloch, Nature 415, 39 (2002).

[24] M. Greiner, O. Mandel, T. W. Hänsch, and I. Bloch, Nature 419, 51 (2002).

[25] T. Kinoshita, T. Wenger, and D. S. Weiss, Nature 440, 900 (2006).

[26] M. Cheneau, P. Barmettler, D. Poletti, M. Endres, P. Schauß, T. Fukuhara, C. Gross, I. Bloch, C. Kollath, and S. Kuhr, Nature 481, 484 (2012).

[27] S. Hofferberth, I. Lesanovsky, T. Schumm, A. Imambekov, V. Gritsev, E. Demler, and J. Schmiedmayer, Nature Physics 4, 489 (2008).

[28] A. Schwarzkopf, R. E. Sapiro, and G. Raithel, Phys. Rev. Lett. 107, 103001 (2011).

[29] R. Löw, H. Weimer, J. Nipper, J. B. Balewski, B. Butscher, H. P. Büchler, and T. Pfau, J. Phys. B: At. Mol. Opt. Phys. 45, 113001 (2012).

[30] M. Saffman, T. G. Walker, and K. Mølmer, Rev. Mod. Phys. 82, 2313 (2010).

[31] N. Malossi, M. M. Valado, S. Scotto, P. Huillery, P. Pillet, D. Ciampini, E. Arimondo, and O. Morsch, Phys. Rev. Lett. 113, 023006 (2014).

[32] C. Carr, R. Ritter, C. G. Wade, C. S. Adams, and K. J. Weatherill, Phys. Rev. Lett. 111, 113901 (2013).

[33] C. S. Hofmann, G. Günter, H. Schempp, M. Robert-deSaint-Vincent, M. Gärttner, J. Evers, S. Whitlock, and M. Weidemüller, Phys. Rev. Lett. 110, 203601 (2013).

[34] H. Weimer, R. Löw, T. Pfau, and H. P. Büchler, Phys. Rev. Lett. 101, 250601 (2008).

[35] T. Pohl, E. Demler, and M. D. Lukin, Phys. Rev. Lett. 104, 043002 (2010).

[36] S. Bettelli, D. Maxwell, T. Fernholz, C. S. Adams, I. Lesanovsky, and C. Ates, Phys. Rev. A 88, 043436 (2013).

[37] P. Schauß, M. Cheneau, M. Endres, T. Fukuhara, S. Hild, A. Omran, T. Pohl, C. Gross, S. Kuhr, and I. Bloch, Nature 491, 87 (2012).

[38] C. Ates, B. Olmos, J. P. Garrahan, and I. Lesanovsky, Phys. Rev. A 85, 043620 (2012).

[39] I. Lesanovsky and J. P. Garrahan, Phys. Rev. Lett. 111, 215305 (2013).

[40] T. E. Lee, H. Häffner, and M. C. Cross, Phys. Rev. Lett. 108, 023602 (2012).

[41] T. E. Lee, H. Häffner, and M. C. Cross, Phys. Rev. A 84, 031402 (2011).

[42] A. Hu, T. E. Lee, and C. W. Clark, Phys. Rev. A 88, 053627 (2013).

[43] J. Jin, D. Rossini, R. Fazio, M. Leib, and M. J. Hartmann, Phys. Rev. Lett. 110, 163605 (2013).

[44] M. Höning, D. Muth, D. Petrosyan, and M. Fleischhauer, Phys. Rev. A 87, 023401 (2013).

[45] T. E. Lee and M. C. Cross, Phys. Rev. A 85, 063822 (2012).

[46] D. W. Schönleber, M. Gärttner, and J. Evers, Phys. Rev. A 89, 033421 (2014).

[47] C. Ates, T. Pohl, T. Pattard, and J. M. Rost, J. Phys. B 39, L233 (2006).

[48] M. Hoening, W. Abdussalam, M. Fleischhauer, and T. Pohl, arXiv:cond-mat/1404.1281 (2014).

[49] D. W. Schönleber, M. Gärttner, and J. Evers, Phys. Rev. A 89, 033421 (2014).

[50] R. Bonifacio and L. A. Lugiato, Phys. Rev. A 18, 1129 
(1978).

[51] G. S. Agarwal and S. R. Shenoy, Phys. Rev. B 25, 1879 (1982).

[52] H. B. Chan and C. Stambaugh, Phys. Rev. Lett. 99, 060601 (2007).

[53] C. Ates, B. Olmos, J. P. Garrahan, and I. Lesanovsky, Phys. Rev. A 85, 043620 (2012).

[54] H. P. Breuer and F. Petruccione, The Theory of Open Quantum Systems (Clarendon Press (Oxford), 2006).

[55] S. M. Barnett and P. M. Radmore, Methods in Theoretical Quantum Optics, Oxford Series in Optical and Imaging Sciences (Clarendon Press (Oxford), 1997).

[56] M. Marinescu, H. R. Sadeghpour, and A. Dalgarno, Phys. Rev. A 49, 982 (1994).

[57] K. Singer, J. Stanojevic, M. Weidemller, and R. Ct, Journal of Physics B: Atomic, Molecular and Optical Physics 38, S295 (2005).

[58] A. W. Glaetzle, M. Dalmonte, R. Nath, I. Rousochatzakis, R. Moessner, and P. Zoller, arXiv:condmat/1404.5326 (2014).
[59] U. Raitzsch, R. Heidemann, H. Weimer, B. Butscher, P. Kollmann, R. Löw, H. P. Büchler, and T. Pfau, New J. Phys. 11, 055014 (2009).

[60] A. Pelissetto and E. Vicari, Phys. Rep. 368, 549 (2002).

[61] Note that, despite the differences in the the experimental, mean-field and phenomenological approaches, which rely on varying either the laser intensity $I$, the Rabi frequency $\Omega$ or the external field $\alpha$ in $V\left(n^{\prime}\right)$, one can still observe the same power law. For example, $I \propto \Omega^{2}$, which implies $\left(I-I_{c}\right)^{-\theta} \propto\left(\Omega^{2}-\Omega_{c}^{2}\right)^{-\theta} \sim 2 \Omega_{c}\left(\Omega-\Omega_{c}\right)^{-\theta}$, with the same exponent $\theta$.

[62] H. K. Janssen, B. Schaub, and B. Schmittmann, Z. Phys. B 73, 539 (1989).

[63] U. C. Täuber, in Ageing and the Glass Transition, Lecture Notes in Physics, Vol. 716, edited by M. Henkel, M. Pleimling, and R. Sanctuary (Springer Berlin Heidelberg, 2007) pp. 295-348.

[64] M. Pleimling, Phys. Rev. B 70, 104401 (2004).

[65] F. Baumann and M. Pleimling, Phys. Rev. B 76, 104422 (2007). 